OPEN ACCESS

Edited by:

Christoph Reiners, University Hospital

Würzburg, Germany

Reviewed by:

Pasqualino Malandrino,

University of Catania, Italy

Daniela Pasquali,

University of Campania Luigi

Vanvitelli, Italy

*Correspondence:

Chengcheng Niu

niuchengcheng@csu.edu.cn

Specialty section:

This article was submitted to

Cancer Endocrinology,

a section of the journal

Frontiers in Endocrinology

Received: 21 February 2020

Accepted: 25 June 2020

Published: 11 August 2020

Citation:

Chen S, Peng Q, Zhang Q and Niu C (2020) Contrast-Enhanced Ultrasound of Primary Squamous Cell Carcinoma of the Thyroid: A Case Report.

Front. Endocrinol. 11:512. doi: 10.3389/fendo.2020.00512

\section{Contrast-Enhanced Ultrasound of Primary Squamous Cell Carcinoma of the Thyroid: A Case Report}

\author{
Sijie Chen ${ }^{1,2}$, Qinghai Peng ${ }^{1,2}$, Qi Zhang ${ }^{1,2}$ and Chengcheng Niu ${ }^{1,2 \star}$ \\ ${ }^{1}$ Department of Ultrasound Diagnosis, The Second Xiangya Hospital, Central South University, Changsha, China, ${ }^{2}$ Research \\ Center of Ultrasonography, The Second Xiangya Hospital, Central South University, Changsha, China
}

Introduction: Primary squamous cell carcinoma of the thyroid (ThyPSCC) is an extremely rare aggressive malignancy with a poor prognosis. However, almost no report thus far has investigated the microvasculature of ThyPSCC imaged using contrast-enhanced ultrasound.

Case Report: A 59-year-old male patient presented to our hospital with progressively worsening hoarse voice symptoms for 20 days and was diagnosed with left unilateral vocal fold palsy. Ultrasonography revealed a solitary marked hypoechoic thyroid nodule with an unclear boundary in the inferior part of the left lobe. Color Doppler flow imaging showed a poor blood flow signal inside this nodule. Contrast-enhanced ultrasound images showed a persistent low peak enhancement of the nodule from its periphery to its center. The time-intensity curve displayed a wash-in time of $10 \mathrm{~s}$, a time to peak of $37 \mathrm{~s}$, a peak signal intensity of $24.5 \%$, and a wash-out time of $70 \mathrm{~s}$ for the thyroid tumor. Finally, left hemithyroidectomy of the thyroid tumor was performed, and histopathologic and immunohistochemical evaluations confirmed the diagnosis of ThyPSCC. Postoperatively, the patient received a combination therapy of chemotherapy, radiotherapy, and targeted therapy, but the patient died 4 months after surgery.

Conclusion: Primary squamous cell carcinoma of the thyroid is a rare but aggressive malignancy of the thyroid. Herein, we reported a case of ThyPSCC and its ultrasonography and pathologic findings.

\footnotetext{
Keywords: thyroid cancer, thyroid nodules (TNs), thyroid ultrasound (US), primary squamous cell carcinoma, contrast enhanced ultrasound (CEUS)
}

\section{INTRODUCTION}

Primary squamous cell carcinoma of the thyroid (ThyPSCC) is a rare thyroid malignancy with high aggressiveness and poor prognosis, comprising $\sim 0.1-1 \%$ of all primary thyroid carcinomas (1-6). Owing to the rapidly progressing and highly invasive nature of the malignancy, patients with ThyPSCC often present at an advanced stage and are difficult to diagnose in the early stage because of its rare incidence and lack of typical imaging findings $(7,8)$.

Thyroid ultrasonography and fine-needle aspiration biopsy (FNAB) are the diagnostic tools of choice for evaluating patients with suspected thyroid nodules (9). Contrast-enhanced ultrasound (CEUS), as a relatively novel US technique, is used to investigate the microvasculature of thyroid nodules and improve the diagnostic accuracy of thyroid nodules accompanied by the use of Thyroid 
Imaging Reporting and Data Systems for ultrasonographic features (10-13). However, very few published studies have reported the use of ultrasonography for ThyPSCC. To our knowledge, this is the first case describing the CEUS findings of ThyPSCC.

\section{CASE REPORT}

A 59-year-old male patient presented to our hospital with progressively worsening hoarse voice symptoms for 20 days and was diagnosed with left unilateral vocal fold palsy. A high-resolution ultrasound instrument (Siemens Acuson S3000, Mountain View, CA, USA) equipped with a 4- to $9-\mathrm{MHz}$ linear probe was used. Thyroid ultrasonography revealed a solitary 3.1 $\times 2.8 \times 2.6-\mathrm{cm}^{3}$ marked hypoechoic thyroid nodule with an unclear boundary in the inferior part of the left lobe (Figure 1A). This nodule exhibited many malignant ultrasound features, such as solid components, hypoechogenicity, and microlobulated margins. Color Doppler flow imaging (CDFI) showed poor blood flow signals in the nodule (Figure 1B). Contrast-enhanced ultrasound was performed with a bolus intravenous injection of $3.0 \mathrm{~mL}$ of SonoVue (Bracco, Milan, Italy) followed by $5 \mathrm{~mL}$ of saline. Contrast pulse sequencing technology was used, and the time-intensity curves (TICs) of the nodule were calculated. The nodule began to be slowly enhanced from the periphery to the center at $10 \mathrm{~s}$ (wash-in time), and the enhancement reached its peak [time to peak (TTP)] at $37 \mathrm{~s}$ with a peak intensity of $24.5 \%$. Then, the nodule slowly declined until all the microbubbles washed out at $70 \mathrm{~s}$ (Figures 1C,D). Based on its malignant conventional ultrasound features and the poor microvasculature revealed by CEUS, we inferred that the nodule was a malignant tumor.

After neck ultrasonography, the positron emission tomography-computed tomography was carried for evaluating the situation of distant metastases. Positron emission tomography-computed tomography showed a mass with increased glucose metabolism in the inferior part of the left thyroid lobe (Figure 2A), which indicated it as a malignant mass, whereas there was no evidence of lymph nodes metastasis and distant metastases. Then, ultrasonography-guided FNAB was performed for the left thyroid mass immediately. Cytologic examination by fine-needle aspiration (FNA) revealed sheets of tumor cells with giant deep-stained nuclei (Bethesda category V) (Figure 2B). Finally, a left hemithyroidectomy of the thyroid tumor was undertaken. The lower edge of the tumor reached the upper mediastinum, and the depth of the tumor invaded the esophagus and trachea, which could not be completely removed. According to the eighth edition of the American Joint Committee on Cancer/Tumor Lymph Node Metastasis (TNM) staging system (14), the patient was in TNM stage III (T4a N0 M0). Histopathological examination of hematoxylin and eosin staining showed that a carcinoma in the inferior
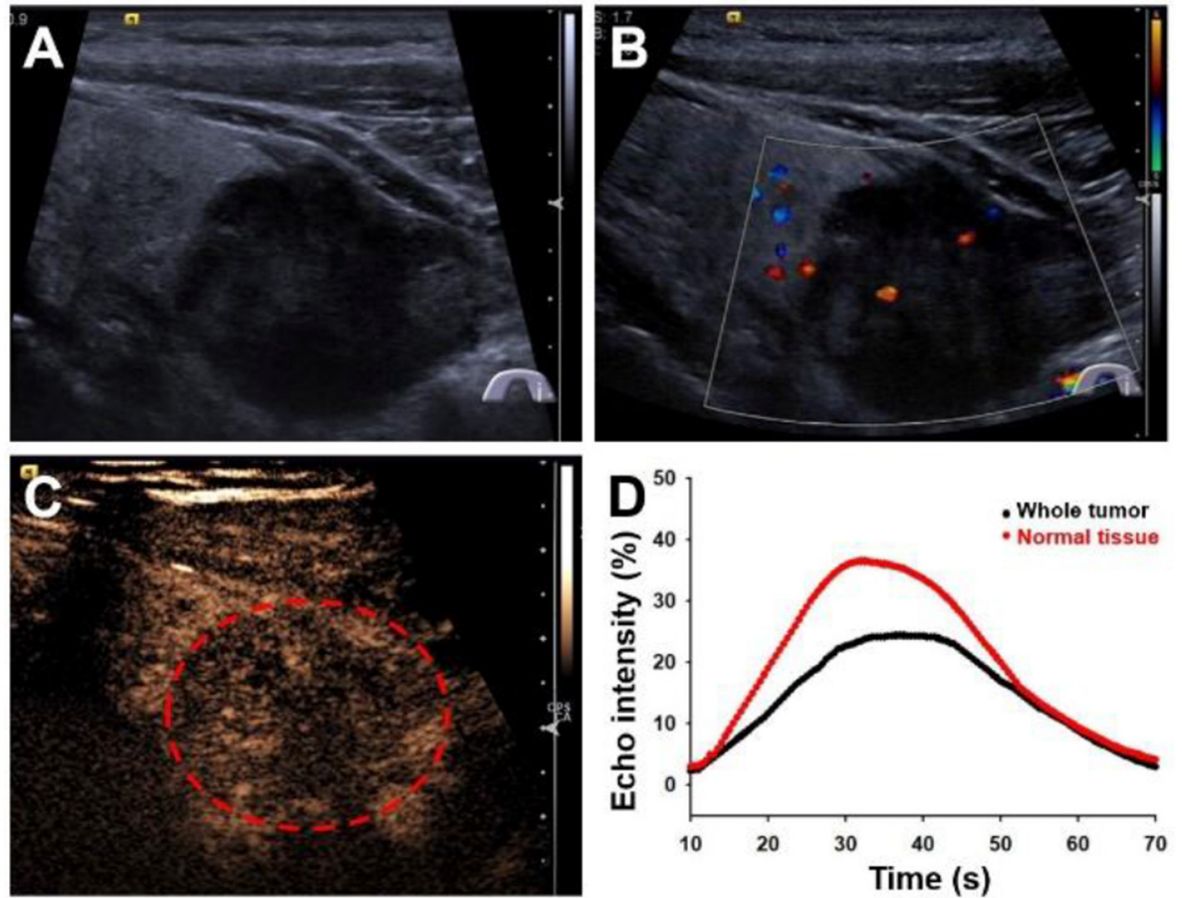

FIGURE 1 | Ultrasonography images of primary squamous cell carcinoma of the thyroid. (A) Longitudinal gray-scale sonography revealed a solid marked hypoechoic thyroid nodule in the inferior part of the left lobe. (B) Color Doppler flow imaging showed a poor blood flow signal inside this nodule. (C) Contrast-enhanced ultrasound image showed a persistent low peak enhancement of the nodule at $37 \mathrm{~s}$. (D) Time-intensity curves displayed the wash-in time of $10 \mathrm{~s}$, TTP of $37 \mathrm{~s}$, peak signal intensity of $24.5 \%$, and wash-out time of 70 s for the thyroid tumor. 

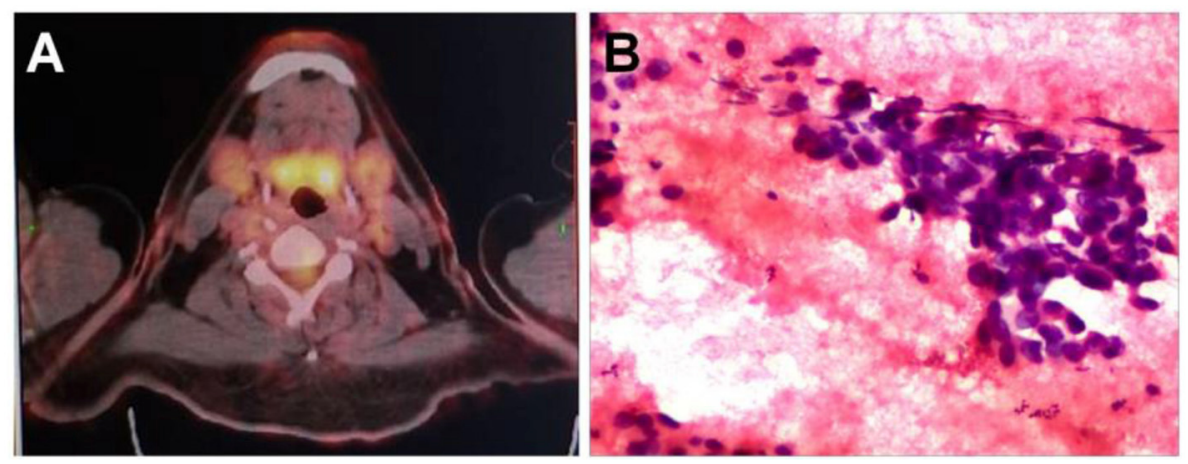

FIGURE 2 | (A) A positron emission tomography-computed tomography scan showed increased ${ }^{18} \mathrm{~F}$-fluorodeoxyglucose metabolism in the left neck mass. (B) Preoperative fine-needle aspiration cytology of the mass demonstrated a few sheets of malignant-looking tumor cells with giant deep stained nuclei (hematoxylin and eosin, magnification $\times 400$ ).
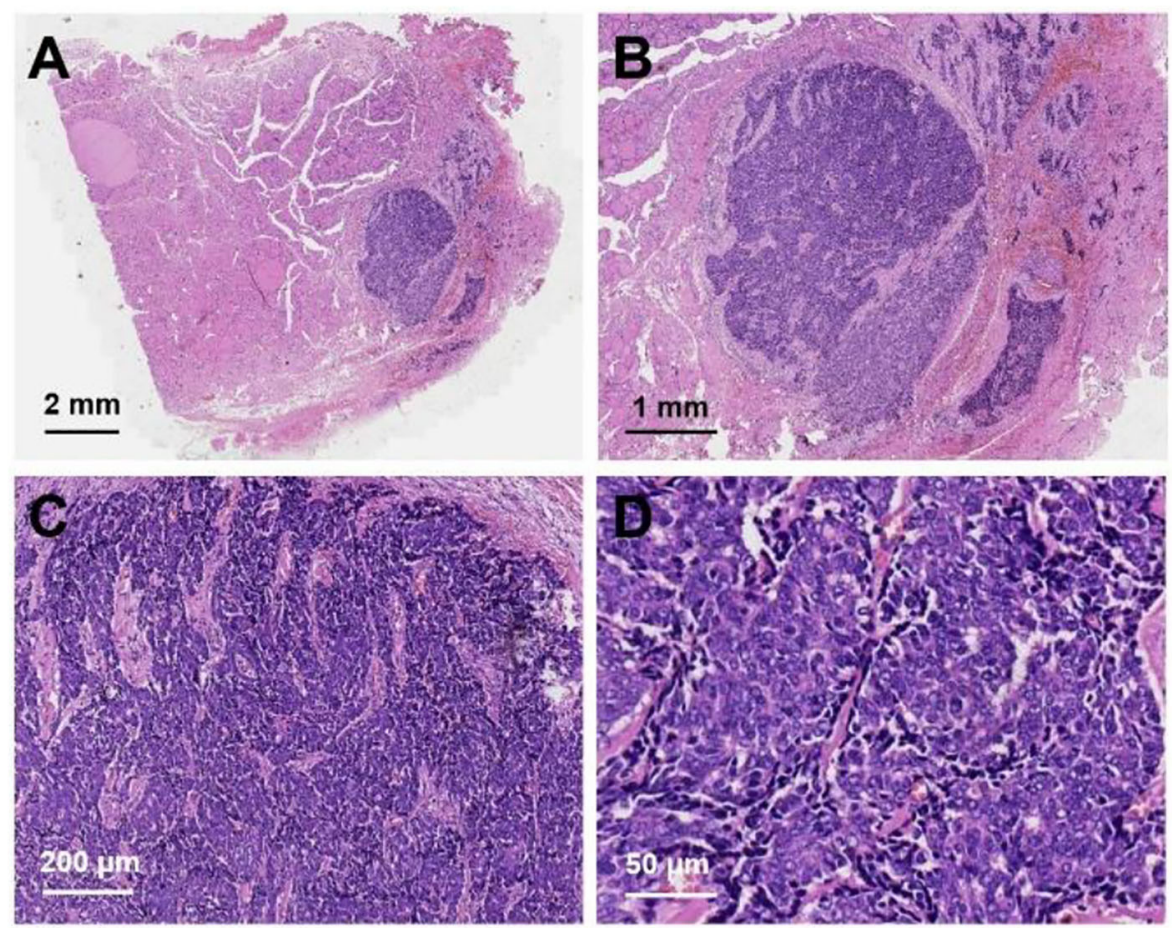

FIGURE 3 | Hematoxylin and eosin staining of primary squamous cell carcinoma of the thyroid: (A) magnification $\times 8$, (B) magnification $\times 20$, (C) magnification $\times$ 100, (D) magnification $\times 400$.

part of the thyroid lobe (Figure 3A) had no obvious palisade arrangement, intercellular bridges, or keratinization with a cancer pearl (Figures 3B-D). Immunohistochemically, tumor cells were positive for cytokeratin 19 (CK19, Figure 4A), cytokeratin 5 and 6 (CK5/6, Figure 4B), epithelial membrane antigen (EMA, Figure 4C), p40 (Figure 4D), p63 (Figure 5A), and Ki-67 (30\%+, Figure 5B) and negative for thyroglobulin (TG, Figure 5C) and thyroid transcription factor 1 (TTF-1, Figure 5D). In view of these findings, the tumor was diagnosed as poorly differentiated ThyPSCC. Postoperatively, the patient received two cycles of chemotherapy with docetaxel/cisplatin, intensity-modulated radiotherapy, and nimotuzumab-targeted therapy. However, the patient died 4 months after surgery.

\section{DISCUSSION}

Primary squamous cell carcinoma of the thyroid is a thyroid malignancy with extremely rare incidence, and the clinical diagnosis and treatment guidelines for this disease have no consensus (4). The biological behavior of ThyPSCC is aggressive, 


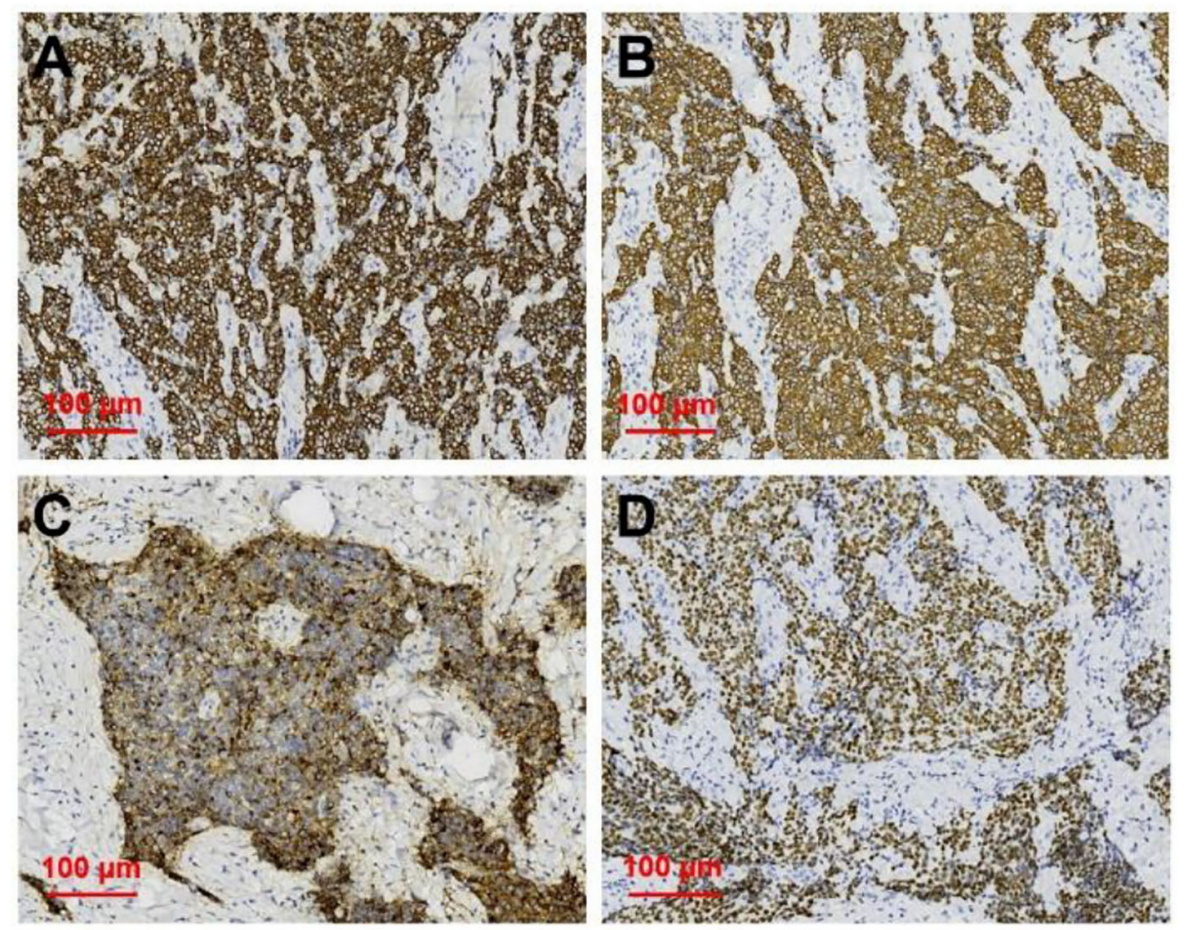

FIGURE 4 | Immunohistochemical staining of primary squamous cell carcinoma of the thyroid (magnification $\times 200$ ). Immunohistochemical staining for (A) CK19, (B) CK5/6, (C) EMA, (D) p40, all of which were deeply stained (positive).
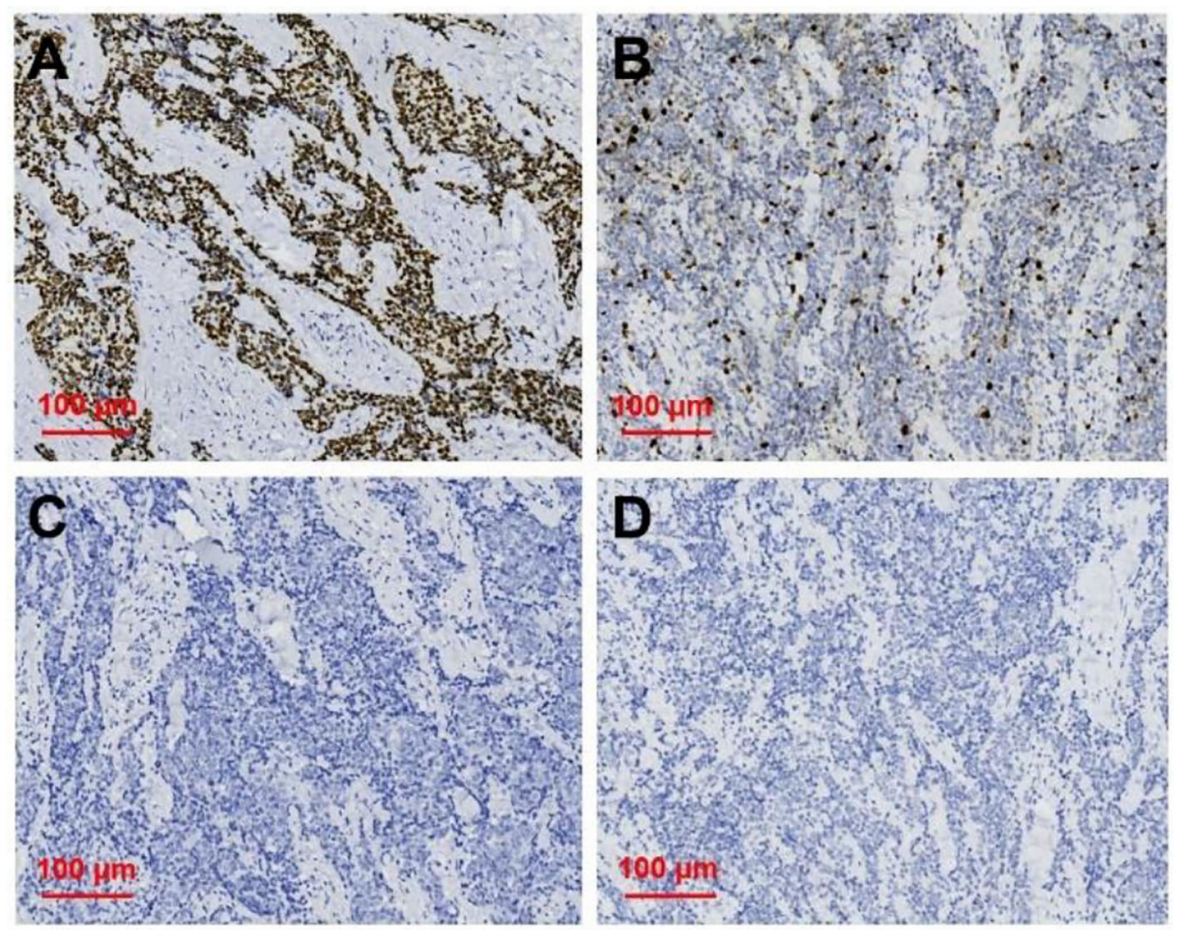

FIGURE 5 | Immunohistochemical staining of primary squamous cell carcinoma of the thyroid (magnification $\times 200)$. Immunohistochemical staining for (A) p63, (B) Ki 67, (C) TG, (D) TTF-1, and p63 was deeply stain (positive); Ki67 proliferation index was 30\%; TG and TTF-1 did not stain (negative). 
and the prognosis is poor, with a median overall survival of 424 months, which depends on the different tumor grades (1). Yang et al. using the Surveillance, Epidemiology, and End Results Program database, reported that poorly differentiated tumor grade occupied the highest percentages of all graded tumors, and the median survival was 4 months, which is similar to the survival time in our case (1).

High-frequency ultrasound, as the basic imaging modality in the diagnosis of thyroid nodules, has found gradually increasing differentiated thyroid cancers over recent years $(15,16)$. The ultrasonography imaging findings of ThyPSCC have seldom been published. Regarding the ultrasonography findings, Chen et al. (17) reported that ThyPSCC presented as a thyroid mass with eggshell calcification, peripheral soft tissue with a blurred margin, and minimal vascular signals on CDFI sonography. In the case of Jang et al. (7), ThyPSCC presented as a large, well-defined, lobulated, heterogeneously hypoechoic mass with diffuse microcalcifications on ultrasonography. Kondo et al. (18) reported that a well-differentiated ThyPSCC showed a cystic hypoechoic mass with a smooth margin and rapidly grew with margin change blurring in 1 year. In our case, this poorly differentiated ThyPSCC presented as a solitary marked hypoechoic thyroid mass with an irregular margin and unclear boundary with a normal thyroid. The irregular margin and unclear boundary with normal thyroid corresponded to tumor invasion with adjacent tissue infiltration, which is consistent with the findings during the operation that tumor invasion with the esophagus cannot be completely removed. Poor blood flow signals on CDFI sonography and persistent hypoenhancement on CEUS of the mass are consistent with squamous cell carcinoma, which has no obvious vascularity on pathologic examination.

Many studies have investigated the application of CEUS to improve the diagnostic accuracy of thyroid nodules, despite its usage in ThyPSCC being scarce. Zhang et al. (15) found that high/circular/equal enhancement indicated benign thyroid nodules, and low enhancement indicated malignant thyroid nodules. Ma et al. (19) investigated whether incomplete, no ring or heterogeneous enhancement, later wash-in time, and low peak intensity on CEUS were independent risk factors in predicting malignant thyroid nodules. Deng et al. (20) detected that papillary thyroid carcinomas (PTCs) exhibited low enhancement, a lower peak signal intensity, and a lower area under the curve (AUC) than peripheral thyroid parenchyma on CEUS $(13,20)$. In our study, the TICs of CEUS for ThyPSCC showed a wash-in time of $10 \mathrm{~s}$, a TTP of $37 \mathrm{~s}$, a peak signal intensity as low as $24.5 \%$, and a wash-out time of $70 \mathrm{~s}$. This is similar to the results of PTCs with a slow wash-in time, a lower peak signal intensity, and a lower AUC, as in previous reports (13). To our knowledge, no reports on CEUS imaging findings of ThyPSCC have appeared in the English-language literature. According to Jang et al. (7), ThyPSCC showed a large heterogeneously enhancing thyroid mass with a large central non-enhancing portion on enhanced CT, which corresponded well with the squamous cell carcinoma portion with a necrotic portion in pathologic staining. Because of the rapid growth of squamous tumor cells, relatively few interstitial blood vessels in tumors were related to the low peak signal intensity and low AUC on CEUS.

With increasing malignancy in squamous cell carcinoma, the typical squamous cell carcinoma findings of intercellular bridges and keratinized cancer pearl can decrease or disappear. Immunohistochemical staining is useful in diagnosing primary thyroid cancer. In this case, positivity for CK5/6 and EMA and negativity for TTF-1 and TG expression predicted squamous cell carcinoma derivation and excluded the possibility of these common tumors (3, 21). Further positivity for p63 and Ki67 expression as poor prognostic markers was associated with its poorly differentiated tumor grade $(7,22)$.

\section{CONCLUSION}

Primary squamous cell carcinoma of the thyroid is an extremely rare tumor, and very few studies describe its ultrasonographic imaging findings. It is difficult to establish a clinical guideline for diagnosis. Our case presents the CEUS features of ThyPSCC, indicating that the TICs of ThyPSCC are similar to the enhancing parameters of PTCs with a slow wash-in time, a lower peak signal intensity, and a lower AUC.

\section{DATA AVAILABILITY STATEMENT}

The datasets generated for this study are available on request to the corresponding author.

\section{ETHICS STATEMENT}

The studies involving human participants were reviewed and approved by the Ethics Committee of Second Xiangya Hospital, Central South University, China. The patients/participants provided their written informed consent to participate in this study. Written informed consent was obtained from the individual(s) for the publication of any potentially identifiable images or data included in this article.

\section{AUTHOR CONTRIBUTIONS}

All authors listed have made a substantial, direct and intellectual contribution to the work, and approved it for publication.

\section{FUNDING}

This project was funded by the National Natural Science Foundation of China (81974267), Hunan Provincial Natural Science Foundation of China (2018JJ2575), and Hunan Provincial Health Commission Research Foundation Project (B2019166). 


\section{REFERENCES}

1. Yang S, Li C, Shi X, Ma B, Xu W, Jiang H, et al. Primary squamous cell carcinoma in the thyroid gland: a population-based analysis using the SEER database. World J Surg. (2019) 43:1249-55. doi: 10.1007/s00268-019-04906-2

2. Limberg J, Ullmann TM, Stefanova D, Finnerty BM, Beninato T, Fahey TJ, et al. Prognostic characteristics of primary squamous cell carcinoma of the thyroid: a national cancer database analysis. World J Surg. (2020) 44:34855. doi: 10.1007/s00268-019-05098-5

3. Koyama S, Fujiwara K, Nosaka K, Fukuhara T, Morisaki T, Miyake $\mathrm{N}$, et al. Immunohistochemical features of primary pure squamous cell carcinoma in the thyroid: an autopsy case. Case Rep Oncol. (2018) 11:41824. doi: 10.1159/000490410

4. Wang SS, Ye DX, Wang B, Xie C. The expressions of keratins and P63 in primary squamous cell carcinoma of the thyroid gland: an application of raman spectroscopy. Onco Targets Ther. (2020) 13:58591. doi: 10.2147/OTT.S229436

5. Yasumatsu R, Sato M, Uchi R, Nakano T, Hashimoto K, Kogo R, et al. The treatment and outcome analysis of primary squamous cell carcinoma of the thyroid. Auris Nasus Larynx. (2018) 45:553-7. doi: 10.1016/j.anl.2017.07.009

6. Kao NH, Tan CS, H, Koh AJ. The utility of immunohistochemistry in differentiating metastatic primary squamous cell carcinoma of the thyroid from a primary lung squamous cell carcinoma. Case Rep Endocrinol. (2019) 2019:1-4. doi: 10.1155/2019/8641267

7. Jang JY, Kwon KW, Kim SW, Youn I. Primary squamous cell carcinoma of thyroid gland with local recurrence: ultrasonographic and computed tomographic findings. Ultrasonography. (2014) 33:143-8. doi: 10.14366/usg.13022

8. Raggio B, Barr J, Ghandour Z, Friedlander P. Primary squamous cell carcinoma of the thyroid. Ochsner J. (2019) 19:2902. doi: 10.31486/toj.18.0002

9. Haugen BR, Alexander EK, Bible KC, Doherty GM, Mandel SJ, Nikiforov YE, et al. 2015 American thyroid association management guidelines for adult patients with thyroid nodules and differentiated thyroid cancer: the American thyroid association guidelines task force on thyroid nodules and differentiated thyroid cancer. Thyroid. (2016) 26:1-133. doi: 10.1089/thy.2015.0020

10. Tessler FN, Middleton WD, Grant EG, Hoang JK, Berland LL, Teefey SA, et al. ACR thyroid imaging, reporting and data system (TI-RADS): white paper of the ACR TI-RADS committee. J Am Coll Radiol. (2017) 14:587-95 doi: 10.1016/j.jacr.2017.01.046

11. Kwak JY, Han KH, Yoon JH, Moon HJ, Son EJ, Park SH, et al. Thyroid imaging reporting and data system for US features of nodules: a step in establishing better stratification of cancer risk. Radiology. (2011) 260:8929. doi: 10.1148/radiol.11110206radiol.11110206

12. Peng Q, Niu C, Zhang Q, Zhang M, Chen S. Mummified thyroid nodules: conventional and contrast-enhanced ultrasound features. J Ultrasound Med. (2018) 38:441-52. doi: 10.1002/jum.14712

13. Peng Q, Niu C, Zhang M, Chen S. Sonographic characteristics of papillary thyroid carcinoma with coexistent hashimoto's thyroiditis: conventional ultrasound, acoustic radiation force impulse imaging and contrast-enhanced ultrasound. Ultrasound Med Biol. (2019) 45:471-80. doi: 10.16/j.ultrasmedbio.2018.10.020

14. Casella C, Ministrini S, Galani A, Mastriale F, Cappelli C, Portolani N. The new TNM staging system for thyroid cancer and the risk of disease downstaging. Front Endocrinol. (2018) 9:541. doi: 10.3389/fendo.2018.00541

15. Zhang Y, Zhou P, Tian SM, Zhao YF, Li JL, Li L. Usefulness of combined use of contrast-enhanced ultrasound and TI-RADS classification for the differentiation of benign from malignant lesions of thyroid nodules. Eur Radiol. (2017) 27:1527-36. doi: 10.1007/s00330-016-4508-y

16. Zhang YZ, Xu T, Gong HY, Li CY, Ye XH, Lin HJ, et al. Application of high-resolution ultrasound, real-time elastography, and contrast-enhanced ultrasound in differentiating solid thyroid nodules. Medicine. (2016) 95:e5329. doi: 10.1097/MD.000000000000532900005792-201611080-00016

17. Chen CY, Tseng HS, Lee CH, Chan PW. Primary squamous cell carcinoma of the thyroid gland with eggshell calcification: sonographic and computed tomographic findings. J Ultrasound Med. (2010) 29:166770. doi: 10.7863 /jum.2010.29.11.1667

18. Kondo T, Matsuyoshi A, Matsuyoshi H, Goto R, Ono K, Honda Y, et al. A case of primary thyroid squamous cell cancer: transformation from benign tumour associated with chronic thyroiditis? BMJ Case Rep. (2009) 2009:bcr10.2008.1137. doi: 10.1136/bcr.10.2008.1137

19. Ma JJ, Ding H, Xu BH, Xu C, Song LJ, Huang BJ, et al. Diagnostic performances of various gray-scale, color doppler, and contrastenhanced ultrasonography findings in predicting malignant thyroid nodules. Thyroid. (2014) 24:355-63. doi: 10.1089/thy.201 3.0150

20. Deng J, Zhou P, Tian SM, Zhang L, Li JL, Qian Y. Comparison of diagnostic efficacy of contrast-enhanced ultrasound, acoustic radiation force impulse imaging, and their combined use in differentiating focal solid thyroid nodules. PLoS ONE. (2014) 9:e90674. doi: 10.1371/journal.pone.0090674PONE-D-13-30329

21. Struller F, Senne M, Falch C, Kirschniak A, Konigsrainer A, Muller S. Primary squamous cell carcinoma of the thyroid: case report and systematic review of the literature. Int J Surg Case Rep. (2017) 37:3640. doi: 10.1016/j.ijscr.2017.06.011

22. Wang W, Ouyang Q, Meng C, Jing L, Li X. Treatment optimization and prognostic considerations for primary squamous cell carcinoma of the thyroid. Gland Surg. (2019) 8:683-90. doi: 10.21037/gs.2019.11.07

Conflict of Interest: The authors declare that the research was conducted in the absence of any commercial or financial relationships that could be construed as a potential conflict of interest.

Copyright (c) 2020 Chen, Peng, Zhang and Niu. This is an open-access article distributed under the terms of the Creative Commons Attribution License (CC BY). The use, distribution or reproduction in other forums is permitted, provided the original author(s) and the copyright owner(s) are credited and that the original publication in this journal is cited, in accordance with accepted academic practice. No use, distribution or reproduction is permitted which does not comply with these terms. 